\title{
DAMAGE IDENTIFICATION IN CONTINUOUS STRUCTURES USING FREE VI- BRATION CHARACTERISTICS
}

\author{
Mohamed Abdel-Mooty
}

Department of Construction and Architectural Engineering, The American University in Caro (mamooty@aucegypt.edu)

\begin{abstract}
The natural frequencies and mode shapes of a vibrating structure are unique to the particular structure and are independent of the type or duration of the dynamic loading. They remain unchanged as long as the physical conditions of the structure are unchanged. Damage, however, causes changes in the physical properties of the structure. The damage is usually localized and the extent of changes in physical properties is usually limited. However, those changes cause alteration to the global dynamic characteristics of the structure presented by its natural frequencies and mode shapes. The use of vibration characteristics as a global measure for damage identification has been recognized as an effective mean to detect the occurrence of damage in structures. While such measure can efficiently recognize damage occurrence, the accurate identification of damage location still largely unsolved issue. Natural frequencies alone cannot identify damage location and one must resort to vibrational modes as spatial characteristics that at least in-principal can locate damage. However, changes in vibration modes due to damage are insignificant and thus their use for damage allocation has been proven inefficient. This research considers examining the differential equation of motion which represents the dynamic equilibrium of the undamaged and damaged structure. Since equilibrium must be maintained at the differential level everywhere, changes due to damage would also causes alteration in the response at the differential level. In this paper closed form expressions of the vibration modes of structures with localized damage are determined using Galerkin's approach. This allows for the examination of the higher derivatives of the vibration modes which reveals that they can be used as effective measures for detecting damage and identifying its location. Damage is modeled by reduction in stiffness over a limited length of the continuous structure. Different damage levels, damage extensions and damage locations are considered. The proposed methodology is applied to non-uniform beams modeling bridge-like structures. Different types and level of damage at different locations and over various length of the beams are considered in the paper. Damage location can be accurately identified using the proposed method as part of a long-term structural health monitoring and non-destructive evaluation scheme.
\end{abstract}

Keywords: Damage detection, Health monitoring, Galerkin's Approach. 


\section{INTRODUCTION}

The free vibration characteristics of a structure, namely natural frequencies and mode shapes of vibrations, can be used as sensitive indicators of its structural integrity. Localized damage and deterioration in the structure alter its dynamic characteristics [1-4]. The shift in the natural frequencies caused by damage and changes in the structural properties is the most commonly used damage detection indicator. However, it cannot provide spatial information about structural changes. Alternatively, changes in the mode shapes, since they are spatially distributed quantities, can be used, at least in principle, for identifying the damage location. However, experimental studies showed that changes in mode shapes due to damage are not statistically significant from the undamaged case [5]. Farrar et al [6] suggested the use of mode shape curvatures as a spatially distributed sensitive indicator to localized damage. This is supported by the fact that higher derivatives of the mode shapes are much more sensitive to small changes in the structure. However, for accurate determination of higher derivatives, mathematical expressions for vibration modes which are sufficiently differentiable must be available. This offers valuable insight into the relationship between damage and mode shapes and their higher derivatives.

Accurate determination of the free vibration characteristics of continuous system is the heart of most dynamic analysis methods used in practice. The powerful modal-analysis method for forced vibrations of continuous system is based on the expansion of the dynamic response in terms of the mode shapes. The derivation of a closed form mathematical expression for the vibration modes of one dimensional continuous system with localized damage is considered in this paper. Galerkin's approach is used where the mode shapes are expressed in terms of a set of simple functions which are easy to manipulate [7]. The accuracy of the method is verified by comparing the results with those of other well-established numerical methods. The possibility of using the derived mode functions and their higher derivatives in identifying the existence of damage and its location is investigated in the paper.

The applicability of using of vibration modes higher derivatives for locating damage in non-uniform system where mathematical expression for the modes are not available is presented in this paper. The free vibration problem of non-uniform steel beam with open web is studied using finite element method. Numerical values for the vibration modes are obtained. The calculated modes are numerically differentiated to obtain first, second, third, and fourth derivatives of the modes of vibration. Different damage types presented by failure of weld lines are introduced at different location for different lengths of damage. The proposed method was able to accurately identify the damage location and extent. 


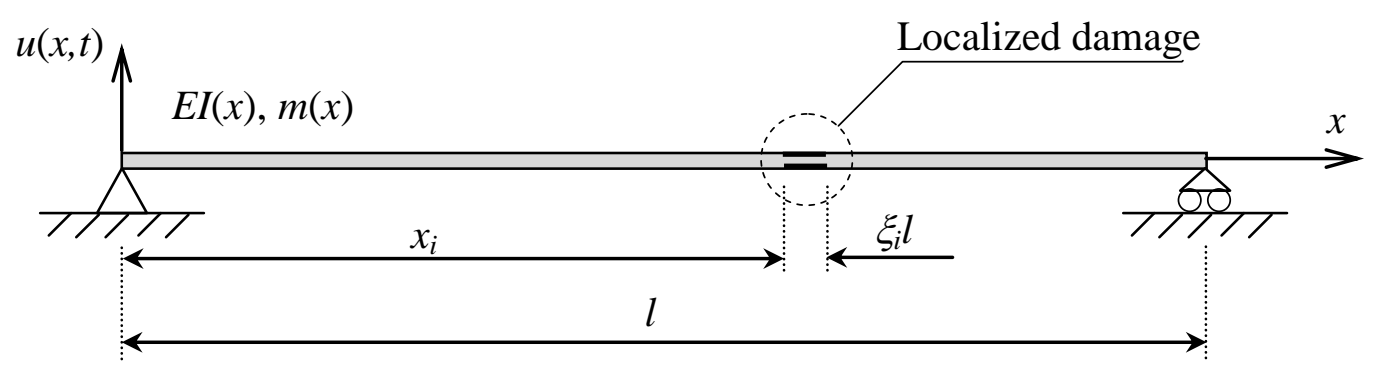

Figure 1. Simply supported elastic beam with variable inertia and mass distribution.

\section{ANALYTICAL MODELING AND CLOSED FORM SOLUTION}

Consider the simply supported beam of length $l$, mass per unit length $m(x)$, and bending stiffness $E I(x)$ shown in Figure 1. The beam may have one or more localized damage at $x_{i}$ over a damaged length of the beam. The damage may be represented by a reduction in the bending stiffness $E I(x)$ over the damaged portion $\xi_{i} l$ of the beam length. The equation of motion for free vibration of the elastic beam of Figure 1 and the corresponding boundary conditions, respectively, read:

$$
\begin{gathered}
\frac{\partial^{2}}{\partial x^{2}} E I(x) \frac{\partial^{2}}{\partial x^{2}} u(x, t)+m(x) \frac{\partial^{2}}{\partial t^{2}} u(x, t)=0, \\
u(0, t)=u(l, t)=\frac{\partial^{2}}{\partial x^{2}} u(0, t)=\frac{\partial^{2}}{\partial x^{2}} u(l, t)=0,
\end{gathered}
$$

where $u(x, t)$ represents the displacement of the beam at any point $x$ and at any time $t$.

In the formulation of Equations (1) and (2), $E I(x)$ and $m(x)$ are the expressions of the bending stiffness and mass distribution along the length of the beam. They are constants for uniform beam with constant elastic properties. For beams with variable cross-section and elastic properties or for uniform beam with localized damage, the length of the beam may be divided into small parts where the cross section and elastic properties in each portion of the beam are assumed constant. The accuracy of the solution depends on the ratio of the average to actual inertia and mass in each portion. The accuracy of the solution can be improved by increasing the divisions of the beam. So, for beam divided into $n_{\alpha}$ parts, each with uniform bending stiffness $\alpha_{i} E I$, and $n_{\beta}$ parts, each of uniform mass $\beta_{j} m$, the stiffness and mass distribution reads

$$
\begin{aligned}
& E I(x)=\alpha_{i} E I, \quad x_{i-1} \leq x \leq x_{i}, \quad 0 \leq \alpha_{i} \leq 1, \quad i=1, n_{\alpha} \\
& m(x)=\beta_{j} m, \quad x_{j-1} \leq x \leq x_{j}, \quad 0 \leq \beta_{j} \leq 1, \quad j=1, n_{\beta}
\end{aligned}
$$

Where $E I$ and $m$ are reference values for the stiffness and mass distribution, and $\alpha_{i}$ and $\beta_{j}$ are factors representing the reduction in stiffness and mass due to damage respectively.

For small vibration and no damping, the following product solution may be assumed for the separation of space distribution and time function: 


$$
u(x, t)=U(x) e^{i \omega t},
$$

Using product solution (5) in (1) and (2) yields the following eigenvalue problem:

$$
\begin{aligned}
& \frac{d^{2}}{d x^{2}} E I(x) \frac{d^{2}}{d x^{2}} U(x)-\omega^{2} m(x) U(x)=0, \\
& U(0)=U(l)=\frac{d^{2}}{d x^{2}} U(0)=\frac{d^{2}}{d x^{2}} U(l)=0,
\end{aligned}
$$

The solution of the eigenvalue problem (6) and (7) yields the natural frequencies $\omega_{k}$ and the corresponding mode shapes of vibrations $U_{k}(x), k=1,2, \ldots$.

Now let the eigenvectors or the vibration modes $U_{k}(x), k=1,2, \ldots$, of the given system be expanded in the following series:

$$
U_{k}(x)=\sum_{j} a_{k j} \Phi_{j}(x)
$$

The functions $\Phi_{j}(x), j=1,2, \ldots$. In (8) must satisfy all the boundary conditions. Using the product solution (6) in the principle of virtual work as

$$
\int_{0}^{l}\left\{\frac{d^{2}}{d x^{2}} E I(x) \frac{d^{2}}{d x^{2}} U_{k}(x)-\omega_{k}^{2} m(x) U_{k}(x)\right\} \delta U_{k}(x) d x=0,
$$

yields the following equation of Galerkin [8] since $a_{k j}$ are arbitrary:

$$
\sum_{j} a_{k j}\left\{\int_{0}^{l}\left\{\frac{d^{2}}{d x^{2}} E I(x) \frac{d^{2}}{d x^{2}} \Phi_{j}(x)-\omega_{k}^{2} m(x) \Phi_{j}(x)\right\} \Phi_{r}(x) d x\right\}=0, \quad \mathrm{r}=1,2, \ldots
$$

Equations (10) can be rewritten as

$$
\sum_{j} a_{k j} \rho_{r j}=0, \quad \quad \mathrm{r}=1,2, \ldots
$$

where

$$
\rho_{r j}=\left\{\int_{0}^{l}\left\{\frac{d^{2}}{d x^{2}} E I(x) \frac{d^{2}}{d x^{2}} \Phi_{j}(x)-\omega_{k}^{2} m(x) U_{k}(x)\right\} \Phi_{r}(x) d x\right\},
$$

A nontrivial solution for Eq. (11) exists only when the determinate of the coefficients vanishes, i.e.

$$
\operatorname{det} \rho_{r j}=0 \text {, }
$$

which yields the natural frequencies $\omega_{k}$ and the corresponding vibration modes $U_{k}(x)$, in terms of the coefficient $a_{k j}, j=1,2,, k=1,2, \ldots$ Let the series expansion functions $\Phi_{j}(x)$ be given by

$$
\Phi_{j}(x)=\sqrt{\frac{2}{l}} \sin \frac{j \pi x}{l}, \quad j=1,2, \ldots
$$

which satisfies all boundary conditions of the simply supported beam. Substituting Eq. (14) in (12) and carrying out the integration yields a mathematical expression for $\rho_{r j}$, in which the natural frequencies $\omega_{k}$ are expressed in terms of non-dimensional frequency parameter $\lambda_{k}$, $k=1,2, \ldots$ 


$$
\lambda_{k}=\omega_{k} m l^{4} / E I, \quad k=1,2, \ldots
$$

The accuracy of the calculated natural frequencies and the obtained mathematical expression for the vibrational modes of the continuous elastic beam is illustrated in a previous publication by the author [9]. In this paper, the method is applied to a locally damaged beam where damage is presented by a variation or reduction in the bending stiffness $E I$ over the damaged length of the beam.

Now, let the beam of Figure 1 be of uniform mass $m$ and uniform bending stiffness $E I$ with a local damage at $x=0.6 l$ and extend over a length $0.1 l$ to $x=0.7 l$. The damage is presented by a reduced bending stiffness $\alpha E I, \alpha<1$, over the damaged length.

The frequency parameters $\lambda_{i}, i=1,2, . .$, for the various modes, are calculated for different level of damage $\alpha$ and compared with the results of finite element model (FEM) as shown in Table 1. The results of the present analysis are in good agreement with the finite element method. However, Galerkin's method used herein is has the advantage of providing mathematical expressions for the modes of vibrations Eq. (10). This allows for obtaining closed form mathematical expressions for the vibration modes $U_{k}(x)$ and its higher derivatives $d U_{\mathrm{k}}(x) / d x$, $d^{2} U_{k}(x) / d x^{2}, d^{3} U_{k}(x) / d x 3$ and $d^{4} U_{k}(x) / d x^{4}$ as follows:

$$
\begin{array}{rr}
U_{k}(x)=\sqrt{\frac{2}{l}} \sum_{j} a_{k j} \sin \frac{j \pi x}{l}, & k=1,2, . . \\
\frac{d U_{k}(x)}{d x}=\sqrt{\frac{2}{l}} \sum_{j} a_{k j} \frac{j \pi}{l} \cos \frac{j \pi x}{l}, & k=1,2, . . \\
\frac{d^{2} U_{k}(x)}{d x^{2}}=-\sqrt{\frac{2}{l}} \sum_{j} a_{k j}\left(\frac{j \pi}{l}\right)^{2} \sin \frac{j \pi x}{l}, & k=1,2, . . \\
\frac{d^{3} U_{k}(x)}{d x^{3}}=-\sqrt{\frac{2}{l}} \sum_{j} a_{k j}\left(\frac{j \pi}{l}\right)^{3} \cos \frac{j \pi x}{l}, & k=1,2, . . \\
\frac{d^{4} U_{k}(x)}{d x^{4}}=\sqrt{\frac{2}{l}} \sum_{j} a_{k j}\left(\frac{j \pi}{l}\right)^{4} \sin \frac{j \pi x}{l}, & k=1,2, . .
\end{array}
$$

The vibration modes and their derivatives given by Egs (16)-(20) can be plotted for further examination.

Table 1: Frequency parameter $\lambda$ for different damage levels.

\begin{tabular}{ccccccc}
\hline \multirow{2}{*}{$\begin{array}{c}\text { Mode } \\
\text { No. }\end{array}$} & \multicolumn{2}{c}{ Damage reduction $\boldsymbol{\alpha = \mathbf { 0 . 8 }}$} & \multicolumn{3}{c}{ Damage reduction $\boldsymbol{\alpha = \mathbf { 0 . 6 }}$} \\
\cline { 2 - 7 } & FEM & Galerkin & Diff.\%. & FEM & Galerkin & Diff.\% \\
\hline $\mathbf{1}$ & 93.7 & 93.741 & 0.04 & 88.237 & 88.292 & 0.06 \\
$\mathbf{2}$ & 1513.7 & 1512.0 & -0.11 & 1456.8 & 1447.8 & -0.62 \\
$\mathbf{3}$ & 7726.6 & 7855.2 & 1.66 & 7726.6 & 7801.4 & 0.97 \\
$\mathbf{4}$ & 24084.2 & 24026.0 & -0.24 & 23274.7 & 22839.0 & -1.87 \\
$\mathbf{5}$ & 57874.7 & 59533.0 & 2.87 & 57874.7 & 57839.0 & -0.06 \\
\hline
\end{tabular}


Figure 2 shows the first mode and its higher derivatives for the case of small damage localized over a length $10 \%$ of the beam length from $0.6 l$ to $0.7 l$. The damage is represented by reduction $20 \%$ of the bending stiffness $E I$ (i.e. $\alpha=0.80$ ) over the damaged length. As anticipated, the variation in the mode shape is insignificant, and thus cannot identify the location of damage. The curvature of the first mode shows slight deviation from the undamaged case in the vicinity of damage. The curves show also smaller deviation elsewhere. Similar effect is observed in the first derivative of the curvature of the mode shapes. However, the fourth derivative of the mode indicates clearly the location of damage.

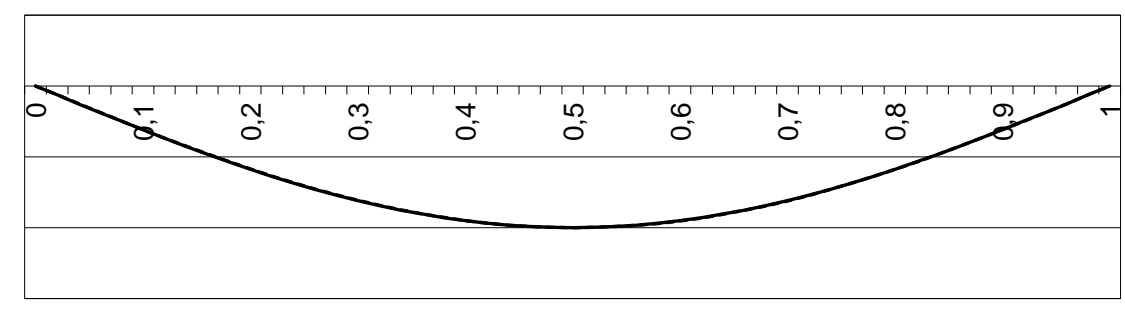

First mode of vibration

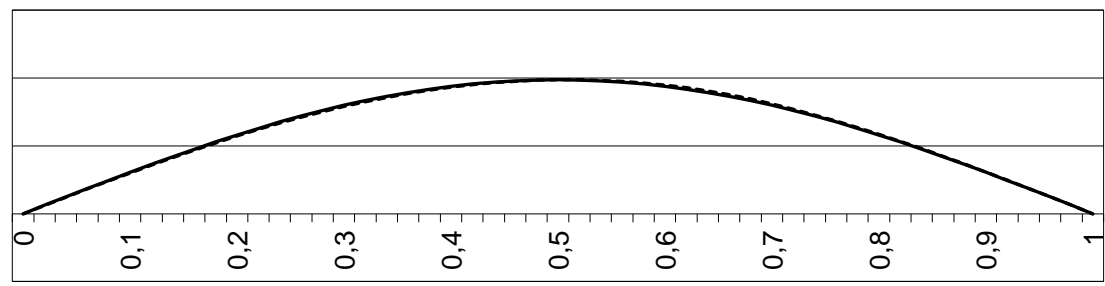

Second derivative of the first mode of vibration

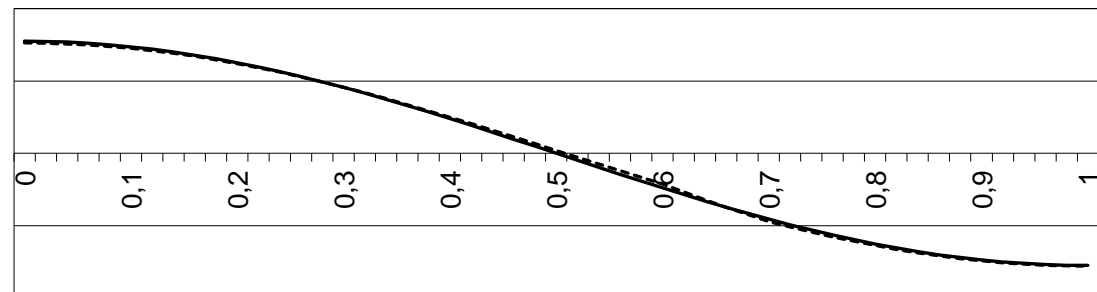

Third derivative of the first mode of vibration

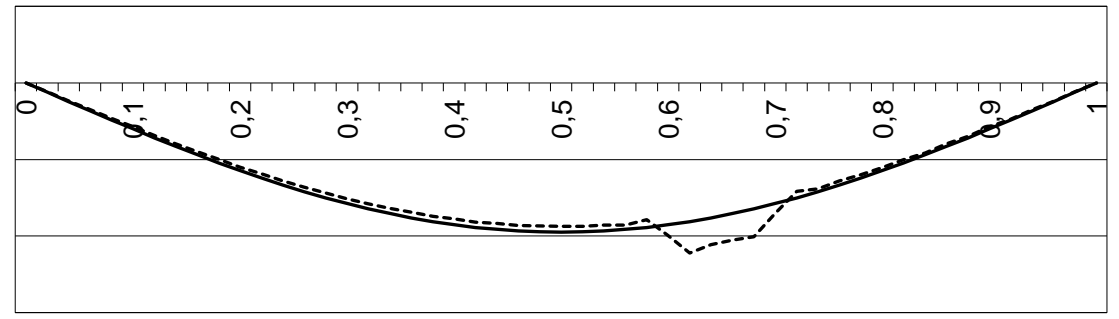

Fourth derivative of the first mode of vibration

Figure 2. First mode of vibration and its derivatives for damaged and undamaged beam — Undamaged beam, - - - - Damaged beam $(\alpha=0.8)$ 


\section{NUMERICAL MODELING OF NON-UNIFORM BEAM WITH LOCAL DAMAGE}

In this section of the paper a non-uniform steel beam with open web is considered. The beam length is $15 \mathrm{~m}$ with web height $500 \mathrm{~mm}$ and thickness $14 \mathrm{~mm}$. The top and bottom flanges are $20 \mathrm{~mm}$ thick and $300 \mathrm{~mm}$ wide each. The beam bending stiffness is increased in the middle $10 \mathrm{~m}$ by providing top and bottom cover plates each of thickness $20 \mathrm{~mm}$ and width $200 \mathrm{~mm}$. The cover plates are attached to the top and bottom flanges through continuous weld lines along their edges. The detailed geometry of the beam is shown in Figure 3.
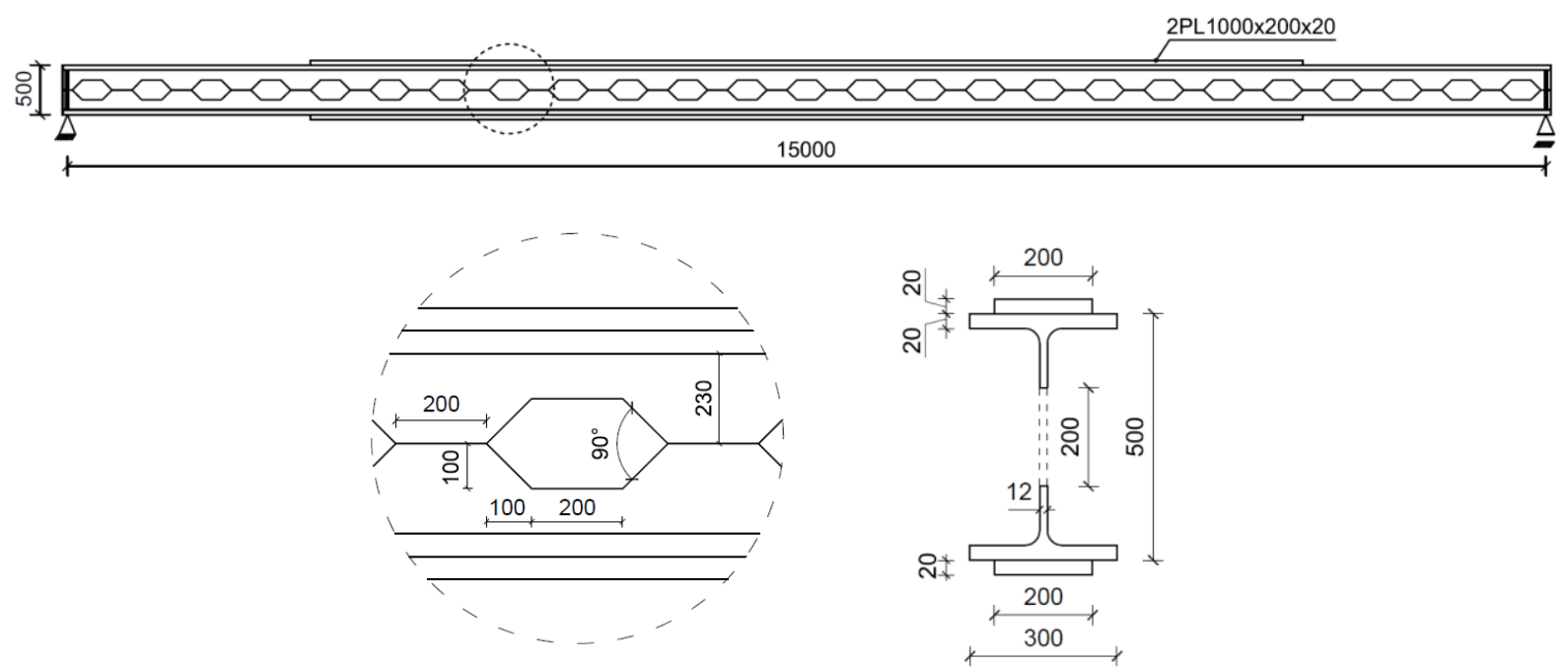

Figure 3. Non-uniform steel beam with open web

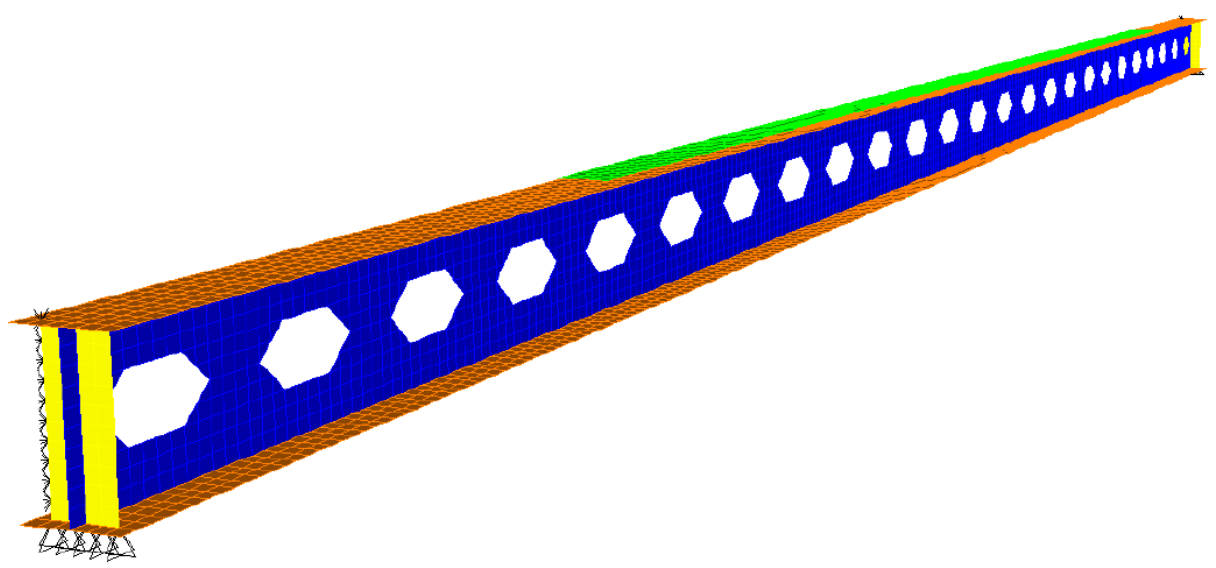

Figure 4. Finite element model using shell element

A finite element model of the beam is constructed using shell elements of size 50x50 $\mathrm{mm}$ for the open web, the top and bottom flange, and the top and bottom cover plate. The weld line between the different plates is modeled by complete restraining the joints at the edge of the two plates. Free vibration analysis of the beam is carried out using SAP2000 commercial structural analysis software. The displacement along the line connecting the top of the web with the center of the top flange is selected to represent the bending modes of vibration of the beam as shown in Figure 5. The first two bending modes for the undamaged beam are calculated as $8.4109 \mathrm{~Hz}$ and $30.647 \mathrm{~Hz}$, respectively. 


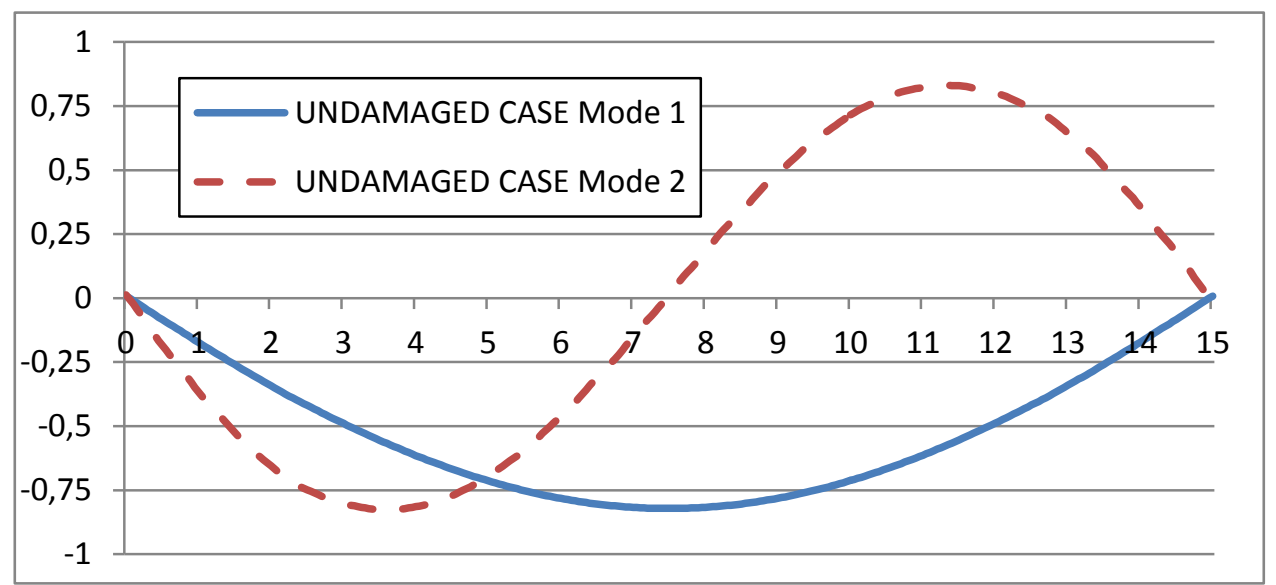

Figure 5. First two bending modes for the undamaged non-uniform open web steel beam

The damage introduced to the beam is represented by failure along the weld line between the different plates. Failure of weld line connecting the cover plates to the flanges is introduced. The extend of weld failure is introduced to the right end of the cover plate over a length equal $500 \mathrm{~mm}, 1000 \mathrm{~mm}$, and $1500 \mathrm{~mm}$. The damage could be on the top cover plate, the bottom cover plate, or both top and bottom cover plates. Other type of damage is introduced along the weld line in the center of the web at different locations.

Table 2. Natural frequencies of the first two bending modes for different damaged cases

\begin{tabular}{llcc}
\hline Case & Damage description & $\begin{array}{c}\text { First Mode } \\
(\mathbf{H z})\end{array}$ & $\begin{array}{c}\text { Second } \\
\text { Mode }(\mathbf{H z})\end{array}$ \\
\hline Undamaged & Undamaged beam & 8.4109 & 30.467 \\
Damage 1 & weld damage at first 0.50 m of top cover plate & 8.3827 & 30.192 \\
Damage 2 & weld damage at first 0.50 m of top \& bottom cover plate & 8.3523 & 29.913 \\
Damage 3 & weld damage at first 1.00 m of top cover plate & 8.3401 & 23.831 \\
Damage 4 & weld damage at first 1.00 m of top \& bottom cover plate & 8.2635 & 16.591 \\
Damage 5 & weld damage at first 1.50 m of top cover plate & 7.0021 & 23.574 \\
Damage 6 & weld damage at first 1.50 m of top \& bottom cover plate & 6.9119 & 16.379 \\
\hline
\end{tabular}

Damage introduces changes in the dynamic characteristics of the structure represented by its natural frequencies and mode shape. As shown in Table 2, changes in the natural frequencies depend on the level and extent of damage. Such alteration in the natural frequencies can be insignificant for small damage. For damage case $1,0.50 \mathrm{~m}$ weld damage only in the top cover plate, the changes in the natural frequencies are $0.3 \%$ and $0.9 \%$ in the first and second mode, respectively. For higher levels of damage, changes in the natural frequencies become more pronounced. However, those changes do not help in identifying the location of damage. 
Damage location can be identified by examining changes in the modes of vibration and their higher derivatives. The numerically calculated mode shapes represented by the deformation along the center of the top flange are numerically differentiated to get the first, the second, the third and fourth derivative as shown in Figures 6-9, respectively, for the first mode. For the considered non-uniform beam, the bending stiffness $E I$ of a section through the web opening is much less than that of a complete section between the openings. Furthermore, sudden and large change in the bending stiffness exists at the beginning of the top and bottom cover plates. As explained earlier such changes in the bending stiffness distribution results in corresponding changes in the higher derivatives of the vibration modes, particularly the fourth derivative. This effect is obvious in Figures 8 and 9. Figures 6-9 shows the first mode and its higher derivative for the undamaged case and the damaged case 1 for small damage only in the top cover plate at its right hand side end. The deviation in the higher derivatives due to such small damage is clearly observed which accurately identify the location of damage.

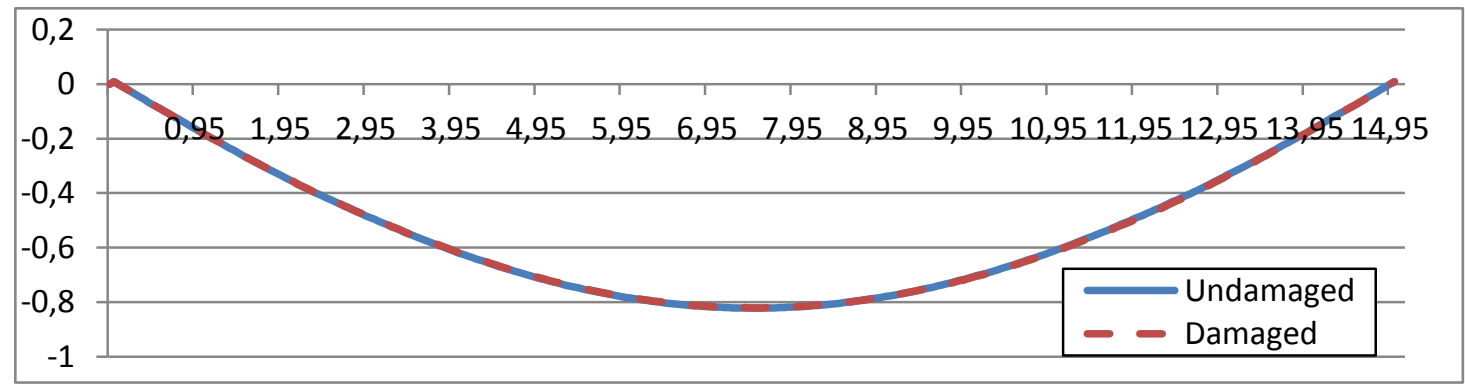

Figure 6. First bending mode for undamaged and damaged case 1.

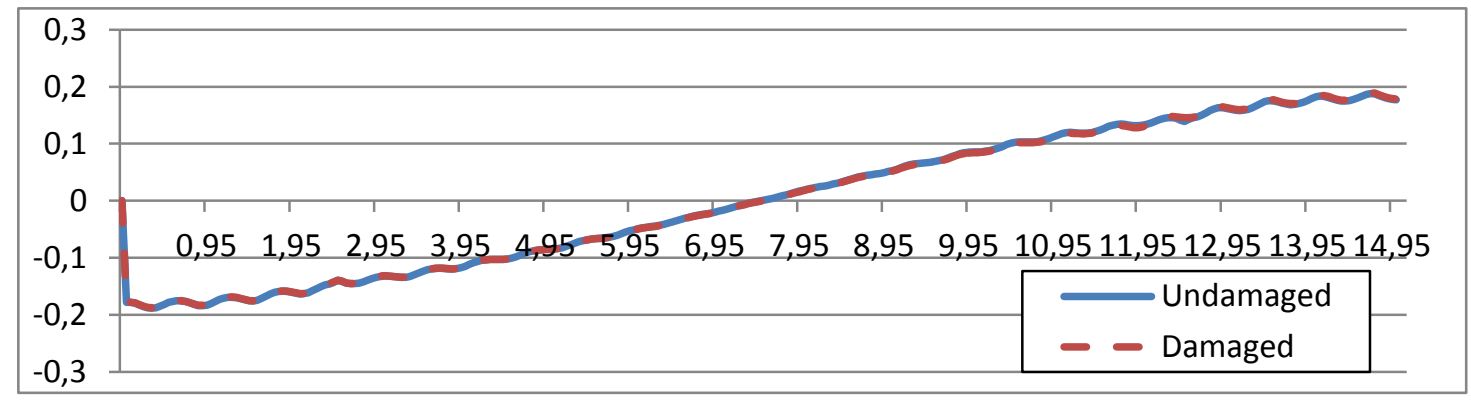

Figure 7. First derivative of the first bending mode for undamaged and damaged case 1.

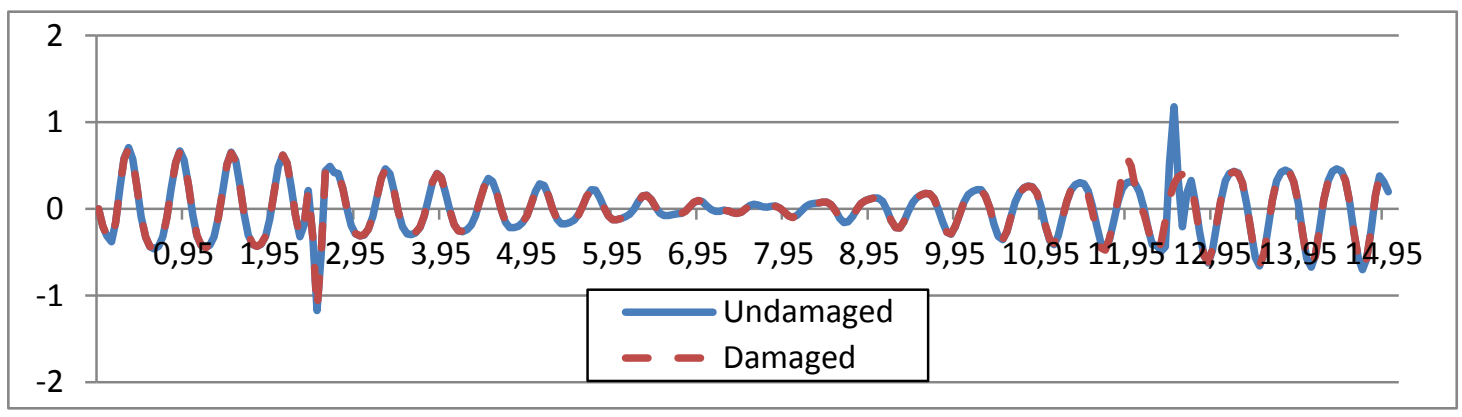

Figure 8 . Second derivative of the first bending mode for undamaged and damaged case 1 


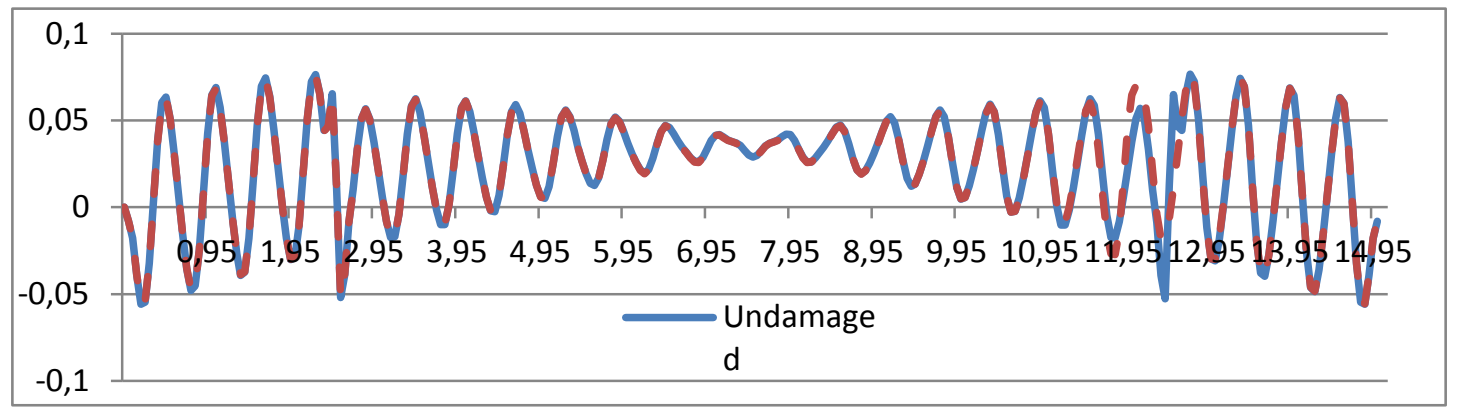

Figure 9. Third derivative of the first bending mode for undamaged and damaged case 1

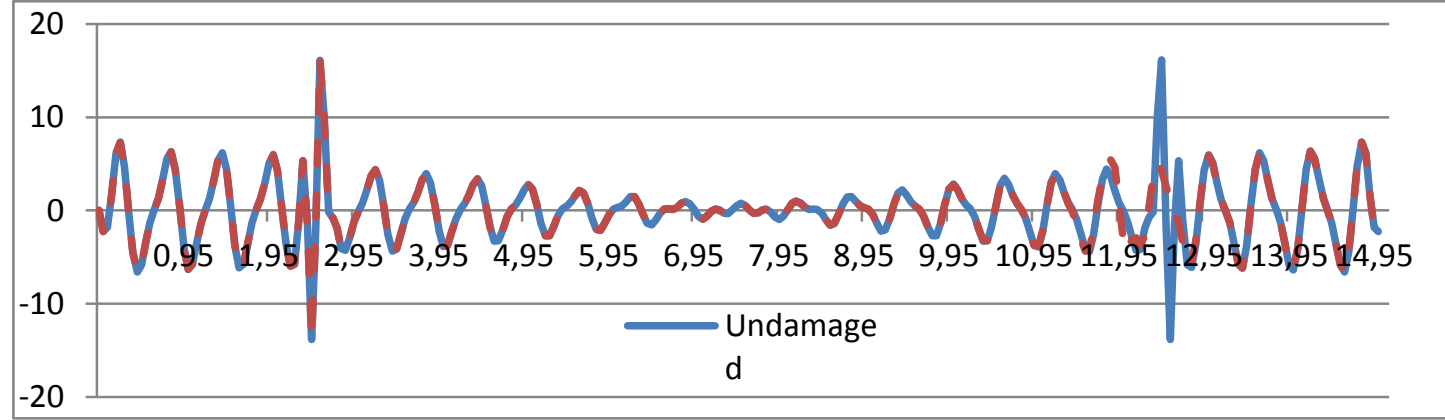

Figure 10. Fourth derivative of the first bending mode for undamaged and damaged case 1.

\section{SUMMARY AND CONCLUSIONS}

The free vibration analysis of beams with local damage is presented in this paper. Local damage over a small portion of the beam is modeled as a local reduction in its bending stiffness $E I(x)$. Simple mathematical expressions for the modes of vibration of damaged beams are obtained using Galerkin's approach. The accuracy of the solution was illustrated by correlating the results to those of the finite element method.

The derived expression for the vibration modes are used to study the effect of localized damage on the free vibration characteristics. Local damage causes small changes in the derived vibration modes which are insignificant, particularly for small damage, to locate the damage. It is found that the curvatures of the derived modes of vibration are more sensitive measures that indicate the damage location when compared with those for the undamaged beam. This is particularly true for high level of damage. The fourth derivative of the vibration mode is very sensitive for changes in the physical condition of the beam due to damage.

Therefore, the fourth derivative of the mode shapes of vibration is the sensitive indicator to use for locating damages. This fact is explained by examining the differential equation of free vibrations. The equation consists of two general terms; the elastic force containing the fourth derivative of deflection (mode) multiplied by $\mathrm{EI}(\mathrm{x})$, and the inertia force containing the mass, frequency and deflection (mode). These two terms must satisfy equilibrium everywhere. Since changes in the modes due to local damage are insignificant, local changes in the 
stiffness $\mathrm{EI}(\mathrm{x})$ must be associated with local changes in the fourth derivative of the mode to maintain equilibrium. This effect clearly locates the position of damage.

The method is examined in a non-uniform open-web steel beam where the modes are calculated numerically using finite element method. The modes are numerically differentiated and plotted for damaged and damaged beams. The correlating the higher derivatives of the vibration modes for the damaged and undamaged cases accurately identifies the location of damage even for small level of damage over limited extent of the beam.

\section{REFERENCES}

[1] Maia, N.M.M and Silva, J.M.M. (Editors) (1997). Theoretical and Experimental Modal Analysis. Research Studies Press Ltd. England.

[2] Doebling, S.W., Farrar, C.R., Prime, M.B. and Shevitz, D.W., "A Review of Damage Identification Methods That Examine Changes in Dynamic Properties", Shock and Vibration Digest, Vol. 30, No. 2, 91-105, 1998.

[3] Abdel-Mooty, M. "Dynamic Modeling and Identification of Discontinuous and Locally Damaged Beams". Proc. 3rd World Conference on Structural Control, 7-12 April 2002, Como, Italy, Fabio Casciati (Ed.), John Wiley \& Sons, Ltd, West Sussex, England, Vol. 3, 679-686, 2002.

[4] Abdel-Mooty, M. and Hashad, A., "Detection of Damage Level and Location in Structures Using Measured Natural Frequencies," Proc.7th Int. Conf. on Computational Structures Technology, Lisbon, Portugal, 7-9 September 2004, B.H.V. Topping and C.A. Mota Soares, (Editors), Civil-Comp Press, Stirling, United Kingdom, paper 245, 2004.

[5] Salawu, S, and Williams, C. (1995). "Bridge Assessment Using Forced Vibration Testing”, Journal of Structural Engineering, ASCE, Vol. 121, No. 2, pp. 161-172.

[6] Farrar, C.R. and Doebling, S.W., "Vibration-Based Damage Detection" In Structural Dynamics@ 2000: Current Status and Future Directions, Ed. D.J. Ewins and D.J. Inman, 145-174, 2001.

[7] Leipholz, H.H.E., "Use of Galerkin's Method for Vibration Problems", Shock and Vibration Digest, Vol. 8, 3-18, 1976.

[8] Leipholz, H.H.E., Direct Variational Methods and Eigenvalue Problems in Engineering, Noordhoff International Publisher, Leyden, 1977.

[9] Abdel-Mooty, M. and Roorda, J., "Free Vibration of Continuous Beams with Singularities in Inertia and Stiffness Distribution". Int. J. Dynamics and Stability of Systems, Vol 6, No. 1, 1-16, 1991 